\title{
ON THE SECOND THEOREM OF CONSISTENCY IN THE THEORY OF ABSOLUTE RIESZ SUMMABILITY
}

BY

B. N. PRASAD AND T. PATI

1.1. Definitions. Let $\sum c_{n}$ be a given infinite series, and $\lambda_{n}$ a positive, steadily increasing monotonic function of $n$, tending to infinity with $n$. We write

$$
C_{\lambda}(\omega)=C_{\lambda}^{0}(\omega)=\sum_{\lambda_{n} \leqq \omega} c_{n}
$$

and

$$
C_{\lambda}^{r}(\omega)=\sum_{\lambda_{n} \leqq \omega}\left(\omega-\lambda_{n}\right)^{r} C_{n}=r \int_{\lambda_{1}}^{\omega} C_{\lambda}(\tau)(\omega-\tau)^{r-1} d \tau \quad(r>0) .
$$

The series $\sum c_{n}$ is said to be summable $(R, \lambda, r), r \geqq 0$, to $C$, if

$$
C_{\lambda}^{r}(\omega) / \omega^{r} \rightarrow C,
$$

as $\omega \rightarrow \infty\left({ }^{1}\right)$.

The series $\sum c_{n}$ is said to be absolutely summable $(R, \lambda, r)$, or summable $|R, \lambda, r|, r \geqq 0$, if

$$
C_{\lambda}^{r}(\omega) / \omega^{r} \in B V(h, \infty)\left({ }^{2}\right),
$$

where $h$ is a finite positive number $\left({ }^{3}\right)$.

1.2. In 1916 Hardy proved the following theorem as an extension of the well-known "second theorem of consistency" for Riesz summability $\left({ }^{4}\right)$, obtained by him and Riesz.

Theorem $\mathrm{A}\left({ }^{5}\right)$. If the series $\sum c_{n}$ is summable $(R, \lambda, \kappa), \kappa \geqq 0$, to the sum $C$, and $\mu$ is a logarithmico-exponential function of $\lambda$, such that

$$
\mu=O\left(\lambda^{\Delta}\right),
$$

where $\Delta i$ a constant, then the series $\sum c_{n}$ is summable $(R, \mu, \kappa)$ to the same sum C.

Received by the editors December 5, 1955, and, in revised form, June 1, 1956.

(1) Riesz [10].

(2) By " $f(x) \in B V(h, k)$ " we mean that $f(x)$ is a function of bounded variation in the interval $(h, k)$; throughout the present paper all infinite intervals are understood to be open on the right.

(3) Obrechkoff [7] and [8].

(4) Hardy and Riesz, [3, pp. 30-33].

(5) Hardy [2]. 
Hirst obtained a generalization $\left(^{6}\right)$ of Hardy's theorem by replacing $\mu$ by a more general function of $\lambda$. Very recently Kuttner has shown that, while Hirst's conditions are both necessary and sufficient in the case in which the order of summability is an integer $\left({ }^{7}\right)$, there do not seem to be available any reasonably simple conditions which are both necessary and sufficient in the case in which the order of summability is nonintegral $\left({ }^{8}\right)$.

In 1942 Chandrasekharan proved $\left({ }^{9}\right)$ the direct analogue of Hardy's theorem for the absolute summability of series by Rieszian means, thus confining the type $\mu$ to a special class of logarithmico-exponential function. Very recently Pati has extended the scope of applicability of the second theorem of consistency for absolutely summable series, when the order of summability is a positive integer, by establishing the following theorem.

THEOREM $\mathrm{B}\left({ }^{10}\right)$. If $\phi(t)$ is a non-negative and monotonic increasing function of $t$ for $t \geqq 0$, steadily tending to infinity as $t$ tends to infinity, such that, for positive integral $\kappa, \phi(t)$ is a $(\kappa+1)$ th indefinite integral for $t \geqq 0$, and

$$
t^{r} \phi^{(r)}(t) / \phi(t) \in B V(h, \infty) \quad(r=1,2, \cdots, \kappa),
$$

where $h$ is a finite positive number, then any infinite series which is summable $\left|R, \lambda_{n}, \kappa\right|$ is also summable $\left|R, \phi\left(\lambda_{n}\right), \kappa\right|$.

The object of the present paper is to establish a parallel theorem in the case in which the order of summability $\kappa$ is positive and nonintegral, and $\phi^{(1)}(t)$ is a monotonic nondecreasing function of $t$.

2.1. We establish the following theorem.

THEOREM. If $\phi(t)$ is a non-negative and monotonic increasing function of $t$ for $t \geqq 0$, steadily tending to infinity as $t$ tends to infinity, such that $\phi^{(1)}(t)$ is monotonic nondecreasing for $t \geqq 0, \phi(t)$ is a $(k+2)$ th indefinite integral for $t \geqq 0$, where $k$ is the integral part of $\kappa\left({ }^{11}\right)$, and

$$
t^{r} \phi^{(r)}(t) / \phi(t) \in B V(h, \infty) \quad(r=1,2, \cdots, k+1),
$$

where $h$ is a finite positive number, then any infinite series which is summable $\left|R, \lambda_{n}, \kappa\right|$, is also summable $\left|R, \phi\left(\lambda_{n}\right), \kappa\right|$.

2.2. It is evident that the truth or otherwise of the theorem depends only upon the behavior of $\phi(t)$ for sufficiently large $t$. We may, therefore, alter $\phi(t)$ in any finite range in any convenient way, and may suppose without any loss of generality that $h=\lambda_{1}$, or even $h=\lambda_{1}=0$, for the sake of con-

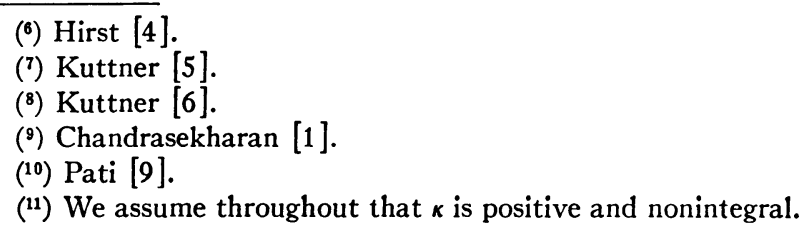


venience, $\phi\left(\lambda_{1}\right)=0$, and that $\phi(t)$ is a $(k+2)$ th indefinite integral for $t \geqq 0$ instead of only for sufficiently large $t$.

2.3. We require the following lemmas for the proof of our theorem.

Lemma $\left.1{ }^{12}\right)$. If $k$ is a positive integer, then

$$
C_{\lambda}(\sigma)=\frac{1}{k !}\left(\frac{d}{d \sigma}\right)^{k} C_{\lambda}^{k}(\sigma) .
$$

Lemma 2. The nth derivative of $\{f(x)\}^{m}$ is a sum of constant multiples of terms of the type

$$
\{f(x)\}^{m-r}\left\{f^{(1)}(x)\right\}^{\alpha_{1}}\left\{f^{(2)}(x)\right\}^{\alpha_{2}} \cdots\left\{f^{(n)}(x)\right\}^{\alpha_{n}},
$$

where $r \leqq n$, and the $\alpha$ 's are positive integers or zeros such that

$$
\sum_{\nu=1}^{n} \alpha_{\nu}=r ; \quad \sum_{\nu=1}^{n} \nu \alpha_{\nu}=n .
$$

Further, if $m$ is a positive integer, then $0<r \leqq m$.

This is a particular case of a result due to Faa di Bruno $\left({ }^{13}\right)$ on the $n$th derivative of a function of a function; the factor $\{f(x)\}^{m-r}$ accrues from the differentiation of $\{f(x)\}^{m}$ with respect to $f(x)$, and is multiplied by a zero factor if $m$ is a positive integer and $r>m$.

Lemma $3\left({ }^{14}\right)$. Let $\phi(t)$ be a non-negative and monotonic increasing function of $t$ for $t \geqq 0$. If $\delta \geqq 0$,

$$
G(\sigma) \in B V(\delta, \infty)
$$

and

$$
\frac{1}{\{\phi(\eta)\}^{r}} \int_{\delta}^{\eta} H(\sigma) d \sigma \in B V(\delta, \infty) \quad(r>0),
$$

then

$$
\frac{1}{\{\phi(\eta)\} r} \int_{\delta}^{\eta} H(\sigma) G(\sigma) d \sigma \in B V(\delta, \infty) .
$$

Lemma 4. If

$$
\chi(\eta)=\int_{0}^{\eta} \sigma^{k+1}\{\phi(\sigma)\} \alpha\left\{\phi^{(1)}(\sigma)\right\}^{\alpha_{1}} \cdots\left\{\phi^{(k+2)}(\sigma)\right\}^{\alpha+2} d \sigma,
$$

(12) Hardy and Riesz [3, p. 31].

(13) C. de la Vallee Poussin [12, p. 89].

(14) Pati [9, Lemma 3]. 
and the $\alpha$ 's are positive integers or zeros such that

$$
0<\alpha+\alpha_{1}+\alpha_{2}+\cdots+\alpha_{k+2}=r \leqq k+1
$$

and

$$
\alpha_{1}+2 \alpha_{2}+\cdots+(k+2) \alpha_{k+2}=k+2,
$$

then, under the hypotheses (2.11) and $h=0$,

$$
\chi(\eta) /\{\phi(\eta)\}^{r} \in B V(0, \infty) .
$$

This follows from a result due to $\operatorname{Pati}\left({ }^{15}\right)$ on making the substitutions: $\kappa=k+1, \lambda_{1}=0$.

Lemma 5. If $\kappa>r \geqq 0$, where $r$ is an integer, and

$$
F(\sigma) \in B V(s, \infty),
$$

then

$$
\frac{1}{\{\phi(\eta)\}^{\kappa}} \int_{0}^{\eta}\{\phi(\eta)-\phi(\sigma)\}^{\kappa-r-1} \phi^{(1)}(\sigma)\{\phi(\sigma)\}^{r} F(\sigma) d \sigma \in B V(s, \infty) .
$$

Proof. Since

$$
\{\phi(\sigma)\} r=\{\phi(\eta)-(\phi(\eta)-\phi(\sigma))\} r,
$$

it suffices to show that, if $F(\sigma) \in B V(S, \infty)$, then

$$
\frac{1}{\{\phi(\eta)\}^{\kappa-r^{\prime}}} \int_{8}^{\eta}\{\phi(\eta)-\phi(\sigma)\}^{\kappa-r^{\prime-1}} \phi^{(1)}(\sigma) F(\sigma) d \sigma \in B V(s, \infty)
$$

where $\kappa>r \geqq r^{\prime} \geqq 0$. Putting $\kappa-r^{\prime}=\delta$, we have to show that

$$
\frac{1}{\{\phi(\eta)\}^{\delta}} \int_{z}^{\eta}\{\phi(\eta)-\phi(\sigma)\}^{\delta-1} \phi^{(1)}(\sigma) F(\sigma) d \sigma \in B V(s, \infty) .
$$

Integrating by parts, we see that the above expression equals

$$
\frac{1}{\delta}\left(1-\frac{\phi(s)}{\phi(\eta)}\right)^{\delta} F(s)+\frac{1}{\delta\{\phi(\eta)\}^{\delta}} \int_{.}^{\eta}\{\phi(\eta)-\phi(\sigma)\}^{\delta} F^{(1)}(\sigma) d \sigma .
$$

Hence, it suffices to show that

$$
\Omega(\eta)=\int_{s}^{\eta}\left(1-\frac{\phi(\sigma)}{\phi(\eta)}\right)^{\delta} F^{(1)}(\sigma) d \sigma \in B V(s . \propto)
$$

Now

(15) Pat: [9 Lemma 4]. 


$$
\begin{aligned}
\int_{s}^{\infty}\left|d_{\eta} \Omega(\eta)\right| & =\int_{s}^{\infty}\left|\int_{s}^{\eta} d_{\eta}\left\{\left(1-\frac{\phi(\sigma)}{\phi(\eta)}\right)^{\delta}\right\} F^{(1)}(\sigma) d \sigma\right| \\
& \leqq \int_{s}^{\infty}\left|F^{(1)}(\sigma)\right| d \sigma \int_{\eta=\sigma}^{\infty} d_{\eta}\left\{\left(1-\frac{\phi(\sigma)}{\phi(\eta)}\right)^{\delta}\right\} \\
& \leqq \int_{s}^{\infty}\left|F^{(1)}(\sigma)\right| d \sigma<\infty,
\end{aligned}
$$

by hypothesis.

Lemma 6. If $\kappa>r \geqq 0$, where $r$ is an integer, and

then

$$
\frac{1}{\{\phi(\eta)\}^{r}} \int_{s}^{\eta} F(\sigma) d \sigma \in B V(s, \infty), \quad s \geqq 0,
$$

$$
\frac{1}{\{\phi(\eta)\}^{r}} \int_{\varepsilon}^{\eta}\left(1-\frac{\phi(\sigma)}{\phi(\eta)}\right)^{\kappa-r} F(\sigma) d \sigma \in B V(s, \infty) .
$$

Proof. Integrating by parts we have

$$
\begin{aligned}
& \frac{1}{\{\phi(\eta)\}^{r}} \int_{s}^{\eta}\left(1-\frac{\phi(\sigma)}{\phi(\eta)}\right){ }^{\kappa-r} F(\sigma) d \sigma \\
& \quad=\frac{(\kappa-r)}{\{\phi(\eta)\}^{\kappa}} \int_{s}^{\eta}\{\phi(\eta)-\phi(\sigma)\}^{\kappa-r-1} \phi^{(1)}(\sigma)\{\phi(\sigma)\}^{r}\left(\frac{1}{\{\phi(\sigma)\}^{r}} \int_{s}^{n} F(\tau) d \tau\right) d \sigma .
\end{aligned}
$$

The result follows by an application of Lemma 5 .

Lemma $7\left({ }^{16}\right)$. If

$$
G(x)=\int_{a}^{x} \xi(x, u) g(u) d u
$$

then

$$
\int_{a}^{\infty}|d G(x)| \leqq \underset{a<u}{\operatorname{upper} \text { bound }}\left\{|\xi(u, u)|+\int_{u}^{\infty}\left|d_{x} \xi(x, u)\right|\right\} \int_{a}^{\infty}|g(u)| d u \text {. }
$$

3.1. Proof of the theorem. By hypothesis

$$
C_{\lambda}^{\kappa}(\eta) / \eta^{\kappa} \in B V(0, \infty)
$$

We have to show that

$$
\frac{1}{\{\phi(\eta)\} \times} \int_{0}^{\eta} C_{\lambda}(\sigma) \frac{d}{d \sigma}\{\phi(\eta)-\phi(\sigma)\} \times d \sigma \in B V(0, \infty),
$$

(16) Tatchell [11, Lemma 1 (i)]. 
that is to say, by Lemma 1 ,

$$
\frac{1}{\{\phi(\eta)\}^{\kappa}} \int_{0}^{\eta}\left(\frac{d}{d \sigma}\right)^{k} C_{\lambda}^{k}(\sigma) \frac{d}{d \sigma}\{\phi(\eta)-\phi(\sigma)\}{ }^{\star} d \sigma \in B V(0, \infty) .
$$

Integrating the integral in the last expression $k$ times by parts, we obtain as the result of integration a constant multiple of

$$
I=\int_{0}^{\eta} C_{\lambda}^{k}(\sigma)\left(\frac{d}{d \sigma}\right)^{k+1}\{\phi(\eta)-\phi(\sigma)\} \star d \sigma .
$$

By Lemma 2, $I$ can be expressed as the sum of constant multiples of integrals of the type

$$
\int_{0}^{\eta} C_{\lambda}^{k}(\sigma)\{\phi(\eta)-\phi(\sigma)\} \alpha-r \prod_{n=1}^{k+1}\left\{\phi^{(n)}(\sigma)\right\}^{\alpha_{n}} d \sigma
$$

where the $\alpha$ 's are positive integers or zeros such that

$$
\alpha_{1}+\alpha_{2}+\cdots+\alpha_{k+1}=r \leqq k+1
$$

and

$$
\alpha_{1}+2 \alpha_{2}+\cdots+(k+1) \alpha_{k+1}=k+1 .
$$

Consider the possibility:

$$
\alpha_{1}+\alpha_{2}+\cdots+\alpha_{k+1}=k+1 .
$$

In this case, since

$$
\alpha_{1}+2 \alpha_{2}+\cdots+(k+1) \alpha_{k+1}=k+1,
$$

by subtraction we get

$$
\alpha_{2}+2 \alpha_{3}+\cdots+k \alpha_{k+1}=0 .
$$

Hence

$$
\begin{gathered}
\alpha_{2}=\alpha_{3}=\cdots=\alpha_{k+1}=0, \\
\alpha_{1}=r=k+1 .
\end{gathered}
$$

Thus $I$ can be expressed as the sum of constant multiples of integrals of the type

$$
I_{1}=\int_{0}^{\eta} C_{\lambda}^{k}(\sigma)\{\phi(\eta)-\phi(\sigma)\}{ }^{\alpha-r} \prod_{n=1}^{k+1}\left\{\phi^{(n)}(\sigma)\right\}{ }^{\alpha_{n}} d \sigma
$$

where

$$
\alpha_{1}+\alpha_{2}+\cdots+\alpha_{k+1}=r<\kappa
$$

and 


$$
\alpha_{1}+2 \alpha_{2}+\cdots+(k+1) \alpha_{k+1}=k+1,
$$

and the integral

$$
I_{2}=\int_{0}^{\eta} C_{\lambda}^{k}(\sigma)\{\phi(\eta)-\phi(\sigma)\}^{\kappa-k-1}\left\{\phi^{(1)}(\sigma)\right\}^{k+1} d \sigma .
$$

We first treat integrals of the type $I_{1}$. Writing

$$
C_{\lambda}^{k}(\sigma)=\frac{1}{k+1} \frac{d}{d \sigma} C_{\lambda}^{k+1}(\sigma),
$$

and integrating by parts, we get integrals of the type

$$
I_{11}=\int_{0}^{\eta} C_{\lambda}^{k+1}(\sigma)\{\phi(\eta)-\phi(\sigma)\}^{\kappa-r-1} \phi^{(1)}(\sigma) \prod_{n=1}^{k+1}\left\{\phi^{(n)}(\sigma)\right\}^{\alpha_{n}} d \sigma
$$

where

$$
\begin{aligned}
\alpha_{1}+\alpha_{2}+\cdots+\alpha_{k+1} & =r<\kappa, \\
\alpha_{1}+2 \alpha_{2}+\cdots+(k+1) \alpha_{k+1} & =k+1
\end{aligned}
$$

and

$$
I_{12}=\int_{0}^{\eta} C_{\lambda}^{k+1}(\sigma)\{\phi(\eta)-\phi(\sigma)\}^{x-r} \prod_{n=1}^{k+2}\left\{\phi^{(n)}(\sigma)\right\}^{\beta_{n}} d \sigma
$$

where

$$
\begin{aligned}
\beta_{1}+\beta_{2}+\cdots+\beta_{k+2} & =r+1<\kappa+1, \\
\beta_{1}+2 \beta_{2}+\cdots+(k+2) \beta_{k+2} & =k+2 .
\end{aligned}
$$

We first prove that

$$
I_{11} /\{\phi(\eta)\}^{\kappa} \in B V(0, \infty) .
$$

We have

$$
\begin{aligned}
& I_{11}=\int_{0}^{\eta}\{\phi(\eta)-\phi(\sigma)\}^{\kappa-r-1} \phi^{(1)}(\sigma)\{\phi(\sigma)\}^{r}\left(\frac{C_{\lambda}^{k+1}(\sigma)}{\sigma^{k+1}}\right)\left(\frac{\sigma \phi^{(1)}(\sigma)}{\phi(\sigma)}\right)^{\alpha_{1}} \ldots \\
& \left(\frac{\sigma^{k+1} \phi^{(k+1)}(\sigma)}{\phi(\sigma)}\right)^{\alpha_{k+1}} d \sigma \\
& =\int_{0}^{\eta}\{\phi(\eta)-\phi(\sigma)\}^{\mathrm{k}-r-1} \phi^{(1)}(\sigma)\{\phi(\sigma)\} r F(\sigma) d \sigma,
\end{aligned}
$$

where $F(\sigma) \in B V(0, \infty)$, by hypotheses (2.11), since $\left.C_{\lambda}^{k+1}(\sigma)\right|_{\sigma} ^{k+1} \in B V(0, \infty)$, by virtue of the first theorem of consistency for absolute Riesz summability.

Hence, using Lemma 5, we obtain 


$$
I_{11} /\{\phi(\eta)\} \ltimes \in B V(0, \infty) .
$$

We next prove that

$$
I_{12} /\{\phi(\eta)\}^{\kappa} \in B V(0, \infty),
$$

that is to say

$$
\frac{1}{\{\phi(\eta)\}^{r}} \int_{0}^{\eta}\left(\frac{C_{\lambda}^{k+1}(\sigma)}{\sigma^{k+1}}\right)\left(1-\frac{\phi(\sigma)}{\phi(\eta)}\right)^{k-r} \sigma^{k+1} \prod_{n=1}^{k+2}\left\{\phi^{(n)}(\sigma)\right\}^{\beta_{n}} d \sigma \in B V(0, \infty) .
$$

By Lemma 4, we have

$$
\frac{1}{\{\phi(\eta)\}^{r}} \int_{0}^{\eta} \sigma^{k+1} \prod_{n=1}^{k+2}\left\{\phi^{(n)}(\sigma)\right\}^{\beta_{n}} d \sigma \in B V(0, \infty)
$$

under the hypotheses (2.11).

Now, since

$$
C_{\lambda}^{k+1}(\sigma) / \sigma^{k+1} \in B V(0, \infty),
$$

we obtain, by Lemma 3,

$$
\frac{1}{\{\phi(\eta)\}^{r}} \int_{0}^{\eta}\left(\frac{C_{\lambda}^{k+1}(\sigma)}{\sigma^{k+1}}\right) \sigma^{k+1} \prod_{n=1}^{k+2}\left\{\phi^{(n)}(\sigma)\right\}^{\beta_{n}} d \sigma \in B V(0, \infty) .
$$

Finally, using Lemma 6, we get the result (3.12).

It remains for us to prove that

$$
\begin{aligned}
I_{2} /\{\phi(\eta)\}^{\kappa} & =\frac{1}{\{\phi(\eta)\}^{*}} \int_{0}^{\eta} C_{\lambda}^{k}(\sigma)\{\phi(\eta)-\phi(\sigma)\}^{k-k-1}\left\{\phi^{(1)}(\sigma)\right\}^{k+1} d \sigma \\
& \in B V(0, \infty) .
\end{aligned}
$$

For $\kappa>0$, we have $\left({ }^{17}\right)$

$$
\begin{aligned}
C_{\lambda}^{k}(\sigma)= & \frac{\Gamma(k+1)}{\Gamma(\kappa+1) \Gamma(k+1-\kappa)} \int_{0}^{\sigma}(\sigma-s)^{k-\kappa} \frac{d}{d s} C_{\lambda}^{\kappa}(s) d s \\
= & \frac{\Gamma(k+1)}{\Gamma(\kappa+1) \Gamma(k+1-\kappa)}\left[-\kappa \int_{0}^{\sigma}\left\{\frac{\partial}{\partial s} \Phi(\sigma, s)\right\} s^{-\kappa} C_{\lambda}^{\kappa}(s) d s\right. \\
& \left.+\int_{0}^{\sigma} \Psi(\sigma, s) \frac{d}{d s}\left\{s^{-\kappa} C_{\lambda}^{\kappa}(s)\right\} d s\right]
\end{aligned}
$$

where

$$
\Phi(\sigma, s)=\int_{s}^{\sigma} u^{\kappa-1}(\sigma-u)^{k-\kappa} d u, \quad \sigma \geqq s
$$

(17) Hardy and Riesz [3, p. 27, Lemma 6]. 


$$
\Psi(\sigma, s)=s^{\kappa}(\sigma-s)^{k-\kappa},
$$

Since, by integration by parts,

$$
-\int_{0}^{\sigma}\left\{\frac{\partial}{\partial s} \Phi(\sigma, s)\right\} s^{-\kappa} C_{\lambda}^{\kappa}(s) d s=\int_{0}^{\sigma} \Phi(\sigma, s) \frac{d}{d s}\left\{s^{-\kappa} C_{\lambda}^{\kappa}(s)\right\} d s,
$$

it follows that

$$
\begin{aligned}
C_{\lambda}^{k}(\sigma)= & \frac{\Gamma(k+1)}{\Gamma(\kappa+1) \Gamma(k+1-\kappa)}\left[\kappa \int_{0}^{\sigma} \Phi(\sigma, s) \frac{d}{d s}\left\{s^{-\kappa} C_{\lambda}^{\kappa}(s)\right\} d s\right. \\
& \left.+\int_{0}^{\sigma} \Psi(\sigma, s) \frac{d}{d s}\left\{s^{-\kappa} C_{\lambda}^{\kappa}(s)\right\} d s\right] .
\end{aligned}
$$

Substituting this expression for $C_{\lambda}^{k}(\sigma)$, we see that we need only prove the following to establish (3.13).

$$
I_{21} /\{\phi(\eta)\} \ltimes \in B V(0, \infty)
$$

and

$$
I_{22} /\{\phi(\eta)\} * \in B V(0, \infty)
$$

where

$$
\left.\begin{array}{c}
I_{21} \\
I_{22}
\end{array}\right\}=\int_{0}^{\eta}\{\phi(\eta)-\phi(\sigma)\}^{\kappa-k-1}\left\{\phi^{(1)}(\sigma)\right\}^{k+1} d \sigma \int_{0}^{\sigma} \Psi(\sigma, s) \frac{d}{d s}\left\{s^{-\kappa} C_{\lambda}^{\kappa}(s)\right\} d s .
$$

Proof of (3.14). Since

$$
\begin{aligned}
& I_{21} /\{\phi(\eta)\}^{\kappa} \\
& =\int_{0}^{\eta} \frac{d}{d s}\left\{s^{-\kappa} C_{\lambda}^{\kappa}(s)\right\} d s \frac{1}{\{\phi(\eta)\}^{\kappa}} \int_{\sigma=s}^{\eta}\{\phi(\eta)-\phi(\sigma)\}^{\kappa-k-1}\left\{\phi^{(1)}(\sigma)\right\}^{k+1} \Phi(\sigma, s) d \sigma,
\end{aligned}
$$

it suffices, by virtue of Lemma 7, to prove only that, uniformly in $s>0$,

$$
\begin{aligned}
g_{1}(\eta, s) & =\frac{1}{\{\phi(\eta)\}^{*}} \int_{\sigma=s}^{\eta}\{\phi(\eta)-\phi(\sigma)\}^{\kappa-k-1}\left\{\phi^{(1)}(\sigma)\right\}^{k+1} \Phi(\sigma, s) d \sigma \\
& \in B V_{\eta}(s, \infty) .
\end{aligned}
$$

Now

$$
\begin{aligned}
\mathfrak{I}_{1}(\eta, s) & =\frac{1}{\{\phi(\eta)\}^{\kappa}} \int_{s}^{\eta}\{\phi(\eta)-\phi(\sigma)\}^{k-k-1} \phi^{(1)}(\sigma)\{\phi(\sigma)\}^{k}\left(\frac{\sigma \phi^{(1)}(\sigma)}{\phi(\sigma)}\right)^{k} \\
& \times\left(\frac{1}{\sigma^{k}} \int_{s}^{\sigma} u^{\kappa-1}(\sigma-u)^{k-\kappa} d u\right) d \sigma,
\end{aligned}
$$

and, therefore, by Lemma 5 and the hypotheses (2.11), it is sufficient for our 
purposes to show that, uniformly in $s>0$,

$$
\frac{1}{\sigma^{k}} \int_{s}^{\sigma} u^{k-1}(\sigma-u)^{k-\alpha} d u \in B V_{\sigma}(s, \infty),
$$

or, what is the same thing,

$$
\int_{s / \sigma}^{1} t^{\kappa-1}(1-t)^{k-\kappa} d t \in B V_{\sigma}(s, \infty) .
$$

We have

$$
\lim _{\sigma \rightarrow \infty} \int_{s / \sigma}^{1} t^{\kappa-1}(1-t)^{k-\kappa} d t=\int_{0}^{1} t^{\kappa-1}(1-t)^{k-\kappa} d t<\infty .
$$

For any $s>0$, and for $\sigma>s$, as $\sigma$ increases, $\int_{s / \sigma}^{1} t^{k-1}(1-t)^{k-\kappa} d t$ increases, and on account of its uniform boundedness in $(s, \infty)$ it is of uniform bounded variation in $(s, \infty)$.

Proof of (3.15). As in the proof of (3.14), it is sufficient, by virtue of Lemma 7 , to prove that, uniformly in $s>0$,

$$
\begin{aligned}
g_{2}(\eta, s) & =\frac{1}{\{\phi(\eta)\}^{\star}} \int_{\delta}^{\eta}\{\phi(\eta)-\phi(\sigma)\}^{k-k-1}\left\{\phi^{(1)}(\sigma)\right\}^{k+1} \Psi(\sigma, s) d \sigma \\
& \in B V_{\eta}(s, \infty) .
\end{aligned}
$$

Proof of (3.17). Since $\Psi(\sigma, s)$ is not defined for $\sigma=s$, we define $g_{2}(s, s)$ as $\lim _{\eta \rightarrow s} \mathscr{S}_{2}(\eta, s)$, which we show below to be finite. Putting $\sigma=s+(\eta-s) v$, we have

$$
\begin{aligned}
\mathscr{g}_{2}(\eta, s) & =\frac{s^{\kappa}}{\{\phi(\eta)\}^{\kappa}} \int_{0}^{1}\left\{\frac{(\eta-s)(1-v)}{\phi(\eta)-\phi(s+(\eta-s) v)}\right\}^{k+1-\kappa} \\
& \times\left\{\phi^{(1)}(s+(\eta-s) v)\right\}^{k+1} v^{k-\kappa}(1-v)^{\kappa-k-1} d v .
\end{aligned}
$$

Now

$$
\lim _{\eta \rightarrow s} \frac{(\eta-s)(1-v)}{\phi(\eta)-\phi(s+(\eta-s) v)}=\frac{1}{\phi^{(1)}(s)} .
$$

Hence

$$
g_{2}(s, s)=\left\{\frac{s \phi^{(1)}(s)}{\phi(s)}\right\}^{\kappa} \int_{0}^{1} v^{k-\kappa}(1-v)^{\kappa-k-1} d v,
$$

which is finite, by hypotheses (2.11), since

$$
\int_{0}^{1} v^{k-\kappa}(1-v)^{\kappa-k-1} d v<\infty .
$$

In order to prove (3.17) we observe that 


$$
\begin{aligned}
\int_{s}^{\infty} \mid & d_{\eta} g_{2}(\eta, s) \mid \leqq \int_{0}^{1} v^{k-\kappa}(1-v)^{\kappa-k-1} d v \\
& \times \int_{s}^{\infty}\left|d_{\eta}\left[s^{\kappa}\left\{\frac{(\eta-s)(1-v)}{\phi(\eta)-\phi(s+(\eta-s) v)}\right\}^{k+1-\kappa} \frac{\left\{\phi^{(1)}(s+(\eta-s) v)\right\} k+1}{\{\phi(\eta)\}^{k}}\right]\right| .
\end{aligned}
$$

Thus, in view of (3.18), it is sufficient for our purpose to show that, uniformly in $0<v<1$ and $s>0$,

$$
s^{*}\left\{\frac{(\eta-s)(1-v)}{\phi(\eta)-\phi(s+(\eta-s) v)}\right\}^{k+1-\kappa} \frac{\left\{\phi^{(1)}(s+(\eta-s) v)\right\}^{k+1}}{\{\phi(\eta)\}^{\kappa}} \in B V_{\eta}(s, \infty) .
$$

Putting $\eta-s=t$, we have only to show that, uniformly in $0<v<1$ and $s>0$,

$$
\begin{aligned}
F(t) & =s^{\kappa}\left\{\frac{(1-v) t}{\phi(s+t)-\phi(s+v t)}\right\}^{k+1-\kappa} \frac{1}{\{\phi(s+t)\}^{\kappa}}\left\{\phi^{(1)}(s+v t)\right\}^{k+1} \\
& \in B V_{t}(0, \infty) .
\end{aligned}
$$

Proof of (3.19). We write $F(t)=U(t) V(t)$, where $V(t)$ is the last factor in the above expression for $F(t)$, and $U(t)$ the rest. Since $\phi^{(1)}(t)$ is nondecreasing, $\{\phi(s+t)-\phi(s+v t)\} /(1-v) t$ is nondecreasing, and hence $U(t)$ is nonincreasing. We also see that $V(t)$ is nondecreasing, and that

$$
\{\phi(s+t)-\phi(s+v t)\} /(1-v) t \geqq \phi^{(1)}(s+v t) .
$$

Therefore, by integration by parts,

$$
\begin{aligned}
\operatorname{Var} F & =\int_{0}^{\infty}\left|U(t) V^{(1)}(t)+V(t) U^{(1)}(t)\right| d t \\
& \leqq \max _{(0, \infty)} F(t)+2 \int_{0}^{\infty} U(t) V^{(1)}(t) d t
\end{aligned}
$$

By (1),

$$
F(t) \leqq s^{\kappa} \frac{\left\{\phi^{(1)}(s+v t)\right\}^{\kappa}}{\{\phi(s+t)\}^{\kappa}} \leqq\left(\frac{s}{s+v t}\right)^{\kappa}\left\{\frac{(s+v t) \phi^{(1)}(s+v t)}{\phi(s+v t)}\right\}^{\kappa} \leqq C,
$$

where $C$ is an absolute finite constant. Now (2) shows that it is sufficient to prove that $W(t)=U(t) V^{(1)}(t)$ has a uniformly bounded integral over $(0, \infty)$. We proceed to prove this.

By virtue of (1),

$$
\begin{aligned}
W(t) & \leqq(k+1) s^{\kappa} v \frac{\left\{\phi^{(1)}(s+v t)\right\}^{\kappa-1}}{\{\phi(s+t)\}^{\kappa}} \phi^{(2)}(s+v t) \\
& \leqq(k+1) s^{\kappa} v \frac{\left\{\phi^{(1)}(s+v t)\right\}^{\kappa-1}}{\{\phi(s+v t)\}^{\kappa}} \phi^{(2)}(s+v t) .
\end{aligned}
$$


Now, for every $T>0$,

$$
\begin{aligned}
\int_{0}^{T} W(t) d t & \leqq \frac{k+1}{\kappa} s^{\kappa} \int_{0}^{T} \frac{d\left\{\phi^{(1)}(s+v t)\right\}^{\kappa}}{\{\phi(s+v t)\}^{\kappa}} \\
& =\left.\frac{k+1}{\kappa}\left\{s \frac{\phi^{(1)}(s+v t)}{\phi(s+v t)}\right\}^{\kappa}\right|_{0} ^{T}+(k+1) v s^{\kappa} \int_{0}^{T} \frac{\left\{\phi^{(1)}(s+v t)\right\}^{\kappa+1}}{\{\phi(s+v t)\}^{\kappa+1}} d t \\
& =T_{1}+T_{2} \text {, say. }
\end{aligned}
$$

We see that $T_{1}$ is uniformly bounded, while

$$
\begin{aligned}
T_{2} & =(k+1) \int_{0}^{T}\left\{\frac{(s+v t) \phi^{(1)}(s+v t)}{\phi(s+v t)}\right\}^{\kappa+1} \frac{s^{\kappa} v d t}{(s+v t)^{\kappa+1}} \\
& \leqq \kappa C \int_{0}^{T} \frac{s^{\kappa} v d t}{(s+v t)^{\kappa+1}} \leqq C,
\end{aligned}
$$

where $C$ is an absolute finite constant.

This completes the proof of the theorem.

\section{REFERENCES}

1. K. Chandrasekharan, The second theorem of consistency for absolutely summable series, J. Indian Math. Soc. (N.S.) vol. 6 (1942) pp. 168-180.

2. G. H. Hardy, The second theorem of consistency for summable series, Proc. London Math. Soc. (2) vol. 15 (1916) pp. 72-88.

3. G. H. Hardy and M. Riesz, The general theory of Dirichlet's series, Cambridge Tracts in Mathematics and Mathematical Physics, No. 18, 1915.

4. K. A. Hirst, On the second theorem of consistency in the theory of summation by typical means, Proc. London Math. Soc. (2) vol. 33 (1932) pp. 353-366.

5. B. Kuttner, Note on the second theorem of consistency for Riesz summability, J. London Math. Soc. vol. 26 (1951) pp. 104-111.

6. - On the "second theorem of consistency" for Riesz summability (II), J. London Math. Soc. vol. 27 (1952) pp. 207-217.

7. N. Obrechkoff, Sur la sommation absolue des séries de Dirichlet, C.R. Acad. Sci. Paris vol. 186 (1928) pp. 215-217.

8. - Über die absolute summierung der Dirichletschen Reihen, Math. Zeit. vol. 30 (1929) pp. 375-386.

9. T. Pati, On the second theorem of consistency in the theory of absolute summability, Quart. J. Math. Oxford Ser. (2) vol. 5 (1954) pp. 161-168.

10. M. Riesz, Sur les séries de Dirichlet et les séries entières, C.R. Acad. Sci. Paris vol. 149 (1909) pp. 909-912.

11. J. B. Tatchell, $A$ theorem on absolute Riesz summability, J. London Math. Soc. vol. 29 (1954) pp. 49-59. 1923.

12. C. de la Vallée Poussin, Course d'analyse infinitésimale, (I), Louvain-Paris, 5th ed.,

The UNiversity,

Allahabad, INdia 